Rev. Bras. Saúde Prod. Anim., Salvador, v.13, n.1, p.62-73 jan/mar, 2012 http://www.rbspa.ufba.br ISSN 15199940

\title{
Classificação multivariada de modelos de crescimento para grupos genéticos de ovinos de corte
}

\author{
Multivariate classification of growth models for lambs genetic groups
}

\author{
SILVEIRA, Fernanda Gomes da ${ }^{1 *}$; SILVA, Fabyano Fonseca $\mathrm{e}^{2}$; \\ CARNEIRO, Paulo Luiz Souza ${ }^{3}$; MALHADO, Carlos Henrique Mendes ${ }^{3}$
}

\footnotetext{
${ }^{1}$ Instituto Federal de Minas Gerais, Bambuí, Departamento de Ciências Exatas, Bambuí, Minas Gerais, Brasil.

${ }^{2}$ Universidade Federal de Viçosa, Centro de Ciências Exatas, Departamento de Estatística, Viçosa, Minas Gerais, Brasil.

${ }^{3}$ Universidade Estadual do Sudoeste da Bahia, Departamento de Ciências Biológicas, Jequié, Bahia, Brasil.

*Endereço para correspondência: fernandaphn@yahoo.com.br
}

\section{RESUMO}

Objetivou-se com a realização deste trabalho, principalmente, utilizar a análise de agrupamento para classificar modelos de crescimento não-lineares tendo em vista os resultados de diferentes avaliadores de qualidade de ajuste ao considerar dados dos seguintes grupos genéticos de ovinos de corte: Dorper x Morada Nova, Dorper x Rabo Largo e Dorper x Santa Inês. Após a indicação do modelo comum adequado aos três grupos, objetivou-se aplicar o teste de identidade de modelos para identificar o grupo genético com maior eficiência de crescimento. Foram realizados ajustes individuais para cada animal, consideraram-se 12 modelos não-lineares, cuja qualidade de ajuste foi medida pelo coeficiente de determinação ajustado, critério de informação de Akaike, critério de informação Bayesiano, erro quadrático médio de predição, coeficiente de determinação de predição e percentual de convergência. A análise de agrupamento permitiu indicar o modelo von Bertalanffy como o mais adequado para descrever as curvas de crescimento dos três grupos genéticos considerados. De acordo com testes de identidade de modelos, o grupo genético Dorper $x$ Santa Inês foi o que apresentou maior peso adulto, portanto, este, o mais recomendado para exploração de carne.

Palavras-chave: análise de agrupamento, Ovis Áries, regressão não-linear, teste de identidade de modelos

\section{SUMMARY}

The main objective of this work was to use the cluster analysis in order to classify nonlinear growth models in relation to different quality fit evaluators when data from the following lambs genetic groups were utilized: Dorper x Morada Nova, Dorper x Rabo Largo e Dorper x Santa Inês. After the choice of the best model, it was also aimed to apply the identity model test in order to identify the most efficient genetic group. The proposed methodology was applied to data of all animals from each group regarding twelve nonlinear models, whose fit quality was measured by determination coefficient, Akaike information criterion, Bayesian information criterion, mean quadratic error of prediction, predicted determination coefficient and convergence percentual. The cluster analysis indicated the von Bertalanffy as the best model for the three data sets. The model identity tests revealed that the Dorper $x$ Santa Inês group presented higher adult weight, therefore this group is recommend for meat production.

Keywords: cluster analysis, model identity test, nonlinear regression, Ovis aries 


\section{INTRODUÇ̃̃O}

Em geral, raças de ovinos de corte naturalizadas do Nordeste brasileiro são adaptadas às condições edafoclimáticas dessa região, e conseguem se desenvolver em condições extremas. Porém, de acordo com Barros et al. (2005), tais raças são pouco produtivas e carecem de precocidade de acabamento e qualidade de carcaça.

Segundo Carneiro et al. (2007), cruzamentos de machos da raça Dorper com ovelhas deslanadas naturalizadas do Nordeste têm sido realizados com o objetivo de conjugar características desejáveis como rusticidade e alta produção. Estas podem ser analisadas por meio de curvas de crescimento (MALHADO et al., 2008).

Modelos de regressão não-linear são adequados para descrever estas curvas, pois apresentam parâmetros interpretáveis biologicamente, como peso à maturidade e taxa de maturidade (GUEDES et al., 2004). Diferentes modelos são apresentados na literatura, mas, informações dadas pelos avaliadores de qualidade de ajuste podem nos indicar qual modelo é o mais apropriado para descrever o crescimento corporal de uma população de ovinos. Contudo, na presença de diferentes populações, os resultados dos avaliadores podem variar de acordo com a população considerada. No melhoramento animal estas populações são designadas por diferentes raças ou combinações de raças, estas denominadas de grupos genéticos.

Além disso, o pesquisador pode comparar os parâmetros das curvas entre as diferentes populações para indicar a população com crescimento mais eficiente. Para tais comparações, designadas como identidade de modelos, um mesmo modelo de regressão deve ser ajustado para todas as populações.
Uma proposta interessante seria utilizar métodos de classificação multivariados, como a análise de agrupamento, que permite a formação de grupos homogêneos de modelos cujos resultados dos avaliadores são concordantes dentro de cada população. E, por meio de medidas de interseção, ficaria fácil a visualização de modelos comuns aos agrupamentos representativos dos melhores ajustes em cada população.

Assim, objetivou-se no presente trabalho, principalmente utilizar a análise de agrupamento para classificar modelos de crescimento não-lineares tendo em vista os resultados de diferentes avaliadores de qualidade de ajuste ao considerar os dados de três diferentes grupos genéticos de ovinos de corte. Após a indicação do modelo comum adequado aos três grupos, objetivou-se também aplicar a identidade de modelos com o intuito de identificar o grupo genético com maior eficiência de crescimento.

\section{MATERIAL E MÉTODOS}

Os dados utilizados neste estudo são provenientes de um experimento conduzido na Estação Experimental de Jaguaquara, pertencente à Empresa Baiana de Desenvolvimento Agrícola S.A., localizada no Município de Jaguaquara, BA, microrregião administrativa de Jequié, BA, de 2003 a 2005.

O rebanho experimental foi composto por 69 matrizes pluríparas: 23 Santa Inês, 23 Morada Nova e 23 Rabo Largo, em condições de reprodução e devidamente selecionadas. Foram utilizados também, dois reprodutores da raça Dorper, de forma que cada reprodutor cobrisse aproximadamente $50 \%$ das matrizes de cada grupo racial. 
As crias acompanharam as matrizes nas pastagens até os 84 dias, quando foram desmamadas.

Estas crias, ou produtos F1, totalizaram 156 animais provenientes dos cruzamentos Dorper x Morada Nova $(\mathrm{DMN})$; Dorper x Rabo Largo (DRL) e Dorper x Santa Inês (DSI). Destes, 36 DMN (26 machos e 10 fêmeas), 61 DRL (36 machos e 25 fêmeas) e 59 DSI (31 machos e 28 fêmeas).

Os produtos $\mathrm{F} 1$, após o desmame, foram mantidos em sistema de produção semi-intensivo, com pastejo durante o dia, em áreas cultivadas com capim Panicum maximum e Brachiaria decumbens, com cobertura de 30 e $70 \%$, respectivamente, com sal mineral comercial à disposição e recolhidos ao final da tarde para o aprisco. Durante o inverno, período mais seco do ano, de junho a outubro, quando há decréscimo na qualidade da pastagem, foi oferecida suplementação com mistura múltipla comercial. Tais animais foram pesados do nascimento até os 210 dias de idade em intervalos de aproximadamente 15 dias.

Os modelos de regressão não-linear apresentados na Tabela 1 foram ajustados aos dados individuais de peso-idade dos ovinos F1 por meio do PROC MODEL do SAS $^{\circledR}$ (SAS INSTITUTE, 2003). Para tal ajuste, utilizou-se o método dos quadrados mínimos ordinários via método iterativo de Gauss-Newton.

Para todos os modelos apresentados na Tabela 1 , o parâmetro $\beta_{1}$ representa o peso adulto, ou peso assintótico, do animal; e o parâmetro $\beta_{3}$ a taxa de maturidade, ou velocidade de crescimento. Os modelos que apresentam o parâmetro $\beta_{4}$ possuem ponto de inflexão variável, cuja localização é determinada pelo parâmetro em questão. Os demais modelos ou apresentam o ponto de inflexão fixo, ou não o possuem, como é o caso dos modelos Brody e Michaelis-
Menten. De forma geral, não há uma interpretação prática para o parâmetro $\beta_{2}$, este uma constante de integração, com exceção do modelo Michaelis-Menten no qual este parâmetro representa a idade na qual se atinge a metade do peso adulto. $\mathrm{Na}$ descrição de tais modelos, ainda é necessário relatar que $\varepsilon_{i}$ representa $\mathrm{o}$ efeito do erro aleatório não observável suposto NIID com média zero e variância desconhecida $\sigma^{2}$. Ressaltamos que as referências de todos os modelos utilizados encontram-se em Silveira (2010).

Foram utilizados seis avaliadores da qualidade de ajuste: coeficiente de determinação ajustado $\left(\mathrm{R}_{\text {aj }}^{2}\right)$, critério de informação de Akaike (AIC), critério de informação bayesiano (BIC), erro quadrático médio de predição (MEP), coeficiente de Determinação de Predição $\left(\mathrm{R}_{\mathrm{p}}^{2}\right)$ e percentual de convergência $(\mathrm{C} \%)$. As expressões destes avaliadores são apresentadas de forma detalhada por Silveira (2010).

Após a obtenção dos avaliadores de qualidade para cada modelo, confeccionaram-se então, conjuntos de dados multivariados, nos quais os modelos correspondem às unidades e os avaliadores às variáveis. Estes foram submetidos à análise de agrupamento no PROC CLUSTER (SAS INSTITUTE, 2003) e, considerado o método centróide. Nesta análise obtiveram-se os valores dos RMSSTD em relação ao número de agrupamentos, a qual gerou o gráfico que disponibilizou a identificação do número ótimo de cluster tendo em vista o método da máxima curvatura (CECON et al., 2008). Como complemento desta última análise, utilizou-se o PROC TREE (SAS INSTITUTE, 2003), para visualizar o dendograma e para verificar quais modelos pertencem aos diferentes grupos obtidos pela discriminação estatística em relação aos avaliadores de qualidade. 
Rev. Bras. Saúde Prod. Anim., Salvador, v.13, n.1, p.62-73 jan/mar, 2012 http://www.rbspa.ufba.br ISSN 15199940

Tabela 1. Modelos de regressão não-linear para descrever curvas de crescimento

\begin{tabular}{|c|c|c|c|}
\hline Modelo & $\begin{array}{l}\text { Modelo de Curva de } \\
\text { Crescimento }\end{array}$ & Modelo & $\begin{array}{l}\text { Modelo de Curva } \\
\text { de Crescimento }\end{array}$ \\
\hline $\begin{array}{c}\text { A } \\
\text { Schnute }\end{array}$ & $\mathrm{y}_{\mathrm{i}}=\frac{\beta_{1}}{\left(1+\beta_{4} \mathrm{e}^{\left(\beta_{3} \beta_{2}-\mathrm{x}_{\mathrm{i}}\right)}\right)^{\frac{1}{\beta_{4}}}}+\mathrm{e}_{\mathrm{i}}$ & $\begin{array}{c}\mathrm{H} \\
\text { Meloun II }\end{array}$ & $y_{i}=\beta_{1}-e^{\left(-\beta_{2}-\beta_{3} x_{i}\right)}+e_{i}$ \\
\hline $\begin{array}{c}\text { B } \\
\text { Mitscherlich }\end{array}$ & $\mathrm{y}_{\mathrm{i}}=\beta_{1}\left(1-\mathrm{e}^{\left(\beta_{3} \beta_{2}-\beta_{3} \mathrm{x}_{\mathrm{i}}\right)}\right)+\mathrm{e}_{\mathrm{i}}$ & $\begin{array}{c}\mathrm{N} \\
\text { Brody }\end{array}$ & $\mathrm{y}_{\mathrm{i}}=\beta_{1}\left(1-\beta_{2} \mathrm{e}^{-\beta_{3} \mathrm{x}_{\mathrm{i}}}\right)+\mathrm{e}_{\mathrm{i}}$ \\
\hline $\begin{array}{c}\text { C } \\
\text { Richards }\end{array}$ & $\mathrm{y}_{\mathrm{i}}=\frac{\beta_{1}}{\left(1+\mathrm{e}^{\left(\beta_{2}-\beta_{3} \mathrm{x}_{\mathrm{i}}\right)}\right)^{\frac{1}{\beta_{4}}}}+\mathrm{e}_{\mathrm{i}}$ & $\begin{array}{c}\text { O } \\
\text { von } \\
\text { Bertalanffy }\end{array}$ & $y_{i}=\beta_{1}\left(1-\beta_{2} e^{-\beta_{3} x_{i}}\right)^{3}+e_{i}$ \\
\hline $\begin{array}{c}\mathrm{D} \\
\text { Gompertz }\end{array}$ & $\mathrm{y}_{\mathrm{i}}=\beta_{1} \mathrm{e}^{\left.\left(-\mathrm{e}^{\left(\beta_{2}-\beta_{3} \mathrm{x}_{\mathrm{i}}\right.}\right)\right)}+\mathrm{e}_{\mathrm{i}}$ & $\begin{array}{c}\mathrm{P} \\
\text { Michaelis- } \\
\text { Menten }\end{array}$ & $\mathrm{y}_{\mathrm{i}}=\frac{\beta_{1} \mathrm{x}_{\mathrm{i}}}{\mathrm{x}_{\mathrm{i}}+\beta_{2}}+\mathrm{e}_{\mathrm{i}}$ \\
\hline $\begin{array}{c}\mathrm{E} \\
\text { Logístico }\end{array}$ & $\mathrm{y}_{\mathrm{i}}=\frac{\beta_{1}}{\left(1+\mathrm{e}^{\left(\beta_{2}-\beta_{3} \mathrm{x}_{\mathrm{i}}\right)}\right)}+\mathrm{e}_{\mathrm{i}}$ & $\begin{array}{l}\text { Q } \\
\text { Michaelis- } \\
\text { Menten } \\
\text { Modificado }\end{array}$ & $\mathrm{y}_{\mathrm{i}}=\frac{\beta_{2} \beta_{3}^{\beta_{4}}+\beta_{1} \mathrm{x}_{\mathrm{i}}^{\beta_{4}}}{\beta_{3}^{\beta_{4}}+\mathrm{x}_{\mathrm{i}}^{\beta_{4}}}+\mathrm{e}_{\mathrm{i}}$ \\
\hline $\begin{array}{c}\text { F } \\
\text { Meloun I }\end{array}$ & $y_{i}=\beta_{1}-\beta_{2} e^{\left(-\beta_{3} x_{i}\right)}+e_{i}$ & $\begin{array}{c}\mathrm{R} \\
\text { Weibull }\end{array}$ & $y_{i}=\beta_{1}-\beta_{2} e^{-e^{\beta_{3}} x_{i}^{\beta_{4}}}+e_{i}$ \\
\hline
\end{tabular}

Posteriormente à escolha do modelo ótimo comum aos três grupos genéticos, aplicou-se o teste de identidade de modelos proposto por Regazzi \& Silva (2004) com o intuito de verificar diferenças nas estimativas dos parâmetros entre os três grupos genéticos avaliados. Para tanto, também se utilizou o PROC MODEL (SAS INSTITUTE, 2003) para ajustar os modelos completo e reduzido, com auxílio de uma variável dummy. O teste em questão foi aplicado separadamente para cada um dos parâmetros de interesse prático $\left(\beta_{1}\right.$ e $\left.\beta_{3}\right)$. Assim, por exemplo, para se testar a hipótese de que o parâmetro $\beta_{1}$ é o mesmo para os três grupos avaliados, considerou-se $\mathrm{H}_{0}^{(1)}: \beta_{11}=\beta_{12}=\beta_{13}$, ao passo que a hipótese alternativa correspondente foi $\mathrm{H}_{\mathrm{a}}^{(1)}$ : nem todos os $\beta_{1}$ 's são iguais.

De acordo com a teoria apresentada por Regazzi \& Silva (2004), e ao se ter como exemplo o modelo von Bertalanffy (Tabela 1), o modelo completo foi dado por:

$$
y_{i}=D_{1}\left[\beta_{11}\left(1-\beta_{21} e^{-\beta_{31} x_{i}}\right)^{3}\right]+D_{2}\left[\beta_{12}\left(1-\beta_{22} e^{-\beta_{32} x_{i}}\right)^{3}\right]+D_{3}\left[\beta_{13}\left(1-\beta_{23} e^{-\beta_{33} x_{i}}\right)^{3}\right]+e_{i} \text {. }
$$


Rev. Bras. Saúde Prod. Anim., Salvador, v.13, n.1, p.62-73 jan/mar, 2012 http://www.rbspa.ufba.br ISSN 15199940

Dessa forma, a hipótese $\mathrm{H}_{0}^{(1)}$ pode ser testada ao assumir o seguinte modelo reduzido:

$$
\mathrm{y}_{\mathrm{i}}=\mathrm{D}_{1}\left[\beta_{1}\left(1-\beta_{21} \mathrm{e}^{-\beta_{31} \mathrm{x}_{\mathrm{i}}}\right)^{3}\right]+\mathrm{D}_{2}\left[\beta_{1}\left(1-\beta_{22} \mathrm{e}^{-\beta_{32} \mathrm{x}_{\mathrm{i}}}\right)^{3}\right]+\mathrm{D}_{3}\left[\beta_{1}\left(1-\beta_{23} \mathrm{e}^{-\beta_{33} \mathrm{x}_{\mathrm{i}}}\right)^{3}\right]+\mathrm{e}_{\mathrm{i}} \text {, }
$$

no qual é assumido um único parâmetro $\beta_{1}$ para representar os três grupos genéticos.

As somas de quadrados dos resíduos provenientes dos ajustes dos modelos (1) e (2), representadas respectivamente por $\mathrm{SQR}_{\Omega}$ e $\mathrm{SQR}_{\omega}$, foram utilizadas para obter a seguinte estatística qui-quadrado: $\chi_{\text {calculado }}^{2}=\mathrm{N} \ln \left(\mathrm{SQR}_{\Omega} /\right.$ $\mathrm{SQR}_{\omega}$ ), sendo $\mathrm{N}$ o número de observações. A regra de decisão consiste em rejeitar $\mathrm{H}_{0}^{(1)}$ a um nível de significância $\alpha$ se $\chi_{\text {calculado }}^{2} \geq \chi_{\alpha}^{2}(v)$, em que $v=p_{\Omega}-p_{\omega}$ é o número de graus de liberdade da estatística do teste, sendo $\mathrm{p}_{\Omega} \mathrm{e}$ $\mathrm{p}_{\omega}$, respectivamente, o número de parâmetros estimados nos modelos completo e reduzido. O mesmo procedimento foi adotado ao se testar a hipótese $\mathrm{H}_{0}^{(3)}: \beta_{31}=\beta_{32}=\beta_{33}$.

\section{RESULTADOS E DISCUSSÃO}

Nas Tabelas 2, 3 e 4 são apresentados, respectivamente para os grupos genéticos Dorper x Morada Nova, Dorper x Rabo Largo e Dorper x Santa Inês, os resultados dos avaliadores da qualidade de ajuste para cada modelo ao se considerar os dados individuais de crescimento.

Tabela 2. Resultados dos avaliadores da qualidade de ajuste para os modelos do grupo genético Dorper x Morada Nova $(\mathrm{DMN})^{1}$

\begin{tabular}{lcccccc}
\hline \multirow{2}{*}{ Modelo } & \multicolumn{6}{c}{ Avaliadores da Qualidade de Ajuste } \\
\cline { 2 - 6 } & $\mathrm{R}_{\text {aj }}^{2}$ & AIC & BIC & MEP & $\mathrm{R}_{\mathrm{p}}^{2}$ & \\
\hline A - Schnute & 0,9536 & 36,4340 & 37,51278 & 2,394103 & 0,993462 & 0,8077 \\
B - Mitscherlich & 0,9692 & 26,9184 & 27,84758 & 0,790985 & 0,998538 & 0,5000 \\
C - Richards & 0,9686 & 17,0443 & 17,44387 & 0,392070 & 0,998744 & 0,2692 \\
D - Gompertz & 0,9743 & 26,1007 & 27,04101 & 0,994654 & 0,997518 & 0,9231 \\
E - Logístico & 0,9732 & 27,3350 & 28,29586 & 1,068450 & 0,997554 & 0,9615 \\
F - Meloun I & 0,9692 & 26,9184 & 27,84756 & 0,783901 & 0,998595 & 0,5000 \\
H - Meloun II & 0,9692 & 26,9184 & 27,84758 & 0,791039 & 0,998538 & 0,5000 \\
N - Brody & 0,9692 & 26,9184 & 27,84756 & 0,791029 & 0,998538 & 0,5000 \\
O - von Bertalanffy & 0,9742 & 25,1104 & 26,06240 & 0,805360 & 0,998532 & 0,8077 \\
P - Michaelis-Menten & 0,9399 & 29,2113 & 29,75984 & 1,757599 & 0,996179 & 0,8077 \\
Q - Michaelis-Menten M. & 0,9712 & 23,4173 & 24,42173 & 0,686830 & 0,998372 & 0,5000 \\
R - Weibull & 0,9730 & 25,2585 & 26,41189 & 0,723404 & 0,998098 & 0,5385 \\
\hline Média & 0,9671 & 26,4654 & 27,36164 & 0,998285 & 0,997722 & 0,6346 \\
Desvio-padrão & 0,0102 & 4,3689 & 4,47531 & 0,546220 & 0,001526 & 0,2160 \\
CV\% & 0,0105 & 0,1651 & 0,16356 & 0,547158 & 0,001530 & 0,3404 \\
\hline
\end{tabular}

${ }^{\mathrm{T}}$ Coeficiente de determinação ajustado $\left(\mathrm{R}^{2}{ }_{\mathrm{aj}}\right)$, critério de informação de Akaike (AIC), critério de informação bayesiano (BIC), erro quadrático médio de predição (MEP), coeficiente de determinação de predição $\left(\mathrm{R}_{\mathrm{p}}^{2}\right)$, percentual de convergência $(\mathrm{C} \%)$ e coeficiente de variação $(\mathrm{CV} \%)$. 
Rev. Bras. Saúde Prod. Anim., Salvador, v.13, n.1, p.62-73 jan/mar, 2012 http://www.rbspa.ufba.br ISSN 15199940

Tabela 3. Resultados dos avaliadores da qualidade de ajuste para os modelos do grupo genético Dorper x Rabo Largo (DRL) ${ }^{1}$

\begin{tabular}{lccccccc}
\hline \multirow{2}{*}{ Modelo } & \multicolumn{7}{c}{ Avaliadores da Qualidade de Ajuste } \\
\cline { 2 - 7 } & \multicolumn{2}{c}{$\mathrm{R}_{\text {aj }}^{2}$} & AIC & BIC & MEP & $\mathrm{R}_{\mathrm{p}}^{2}$ & \\
\hline A - Schnute & 0,9407 & 35,0426 & 37,01784 & 3,173518 & 0,991753 & 0,9375 \\
B - Mitscherlich & 0,9540 & 35,2852 & 36,45601 & 1,258653 & 0,997605 & 0,5833 \\
C - Richards & 0,9725 & 25,5717 & 26,63524 & 1,283962 & 0,995138 & 0,2708 \\
D - Gompertz & 0,9634 & 30,6113 & 31,64892 & 1,280066 & 0,996823 & 0,8125 \\
E - Logístico & 0,9662 & 27,6127 & 28,51031 & 1,306261 & 0,996355 & 0,9583 \\
F - Meloun I & 0,9540 & 35,2852 & 36,45601 & 1,258430 & 0,997606 & 0,5833 \\
H - Meloun II & 0,9540 & 35,2852 & 36,45601 & 1,258369 & 0,997606 & 0,5833 \\
N - Brody & 0,9540 & 35,2852 & 36,45601 & 1,258447 & 0,997606 & 0,5833 \\
O - von Bertalanffy & 0,9615 & 33,1841 & 34,27488 & 1,215016 & 0,997089 & 0,7292 \\
P - Michaelis-Menten & 0,9227 & 36,6163 & 37,28893 & 1,991485 & 0,994133 & 0,8542 \\
Q - Michaelis-Menten M. & 0,9509 & 36,6708 & 38,13961 & 1,401599 & 0,996746 & 0,2917 \\
R - Weibull & 0,9659 & 34,3665 & 35,72301 & 2,004183 & 0,996682 & 0,3125 \\
\hline Média & 0,9550 & 33,4014 & 34,58857 & 1,557499 & 0,996262 & 0,6250 \\
Desvio-padrão & 0,0132 & 3,5864 & 3,69250 & 0,581031 & 0,001778 & 0,2428 \\
CV\% & 0,0139 & 0,1074 & 0,10675 & 0,373054 & 0,001784 & 0,3885 \\
\hline
\end{tabular}

Tabela 4. Resultados dos avaliadores da qualidade de ajuste para os modelos do grupo genético Dorper x Santa Inês (DSI) ${ }^{1}$

\begin{tabular}{lcccccc}
\hline \multirow{2}{*}{ Modelo } & \multicolumn{6}{c}{ Avaliadores da Qualidade de Ajuste } \\
\cline { 2 - 6 } & $\mathrm{R}_{\text {aj }}^{2}$ & AIC & BIC & MEP & $\mathrm{R}_{\mathrm{p}}^{2}$ & \\
\hline A - Schnute & 0,9317 & 42,6032 & 43,61784 & 5,689999 & 0,991521 & 0,9512 \\
B - Mitscherlich & 0,9661 & 31,0505 & 32,12904 & 1,470024 & 0,997775 & 0,6098 \\
C - Richards & 0,9263 & 43,8125 & 45,81500 & 3,191846 & 0,996777 & 0,3415 \\
D - Gompertz & 0,9796 & 27,8202 & 28,81723 & 1,061648 & 0,997784 & 0,9756 \\
E - Logístico & 0,9776 & 29,2923 & 30,29432 & 1,525167 & 0,997177 & 1,0000 \\
F - Meloun I & 0,9709 & 31,2192 & 32,31890 & 2,797085 & 0,998027 & 0,5854 \\
H - Meloun II & 0,9661 & 31,0505 & 32,12904 & 1,478020 & 0,997753 & 0,6098 \\
N - Brody & 0,9661 & 31,0505 & 32,12904 & 1,478199 & 0,997753 & 0,6098 \\
O - von Bertalanffy & 0,9782 & 24,8735 & 25,80715 & 1,060680 & 0,997778 & 0,8537 \\
P - Michaelis-Menten & 0,9053 & 39,4480 & 40,13553 & 3,290704 & 0,994763 & 0,8780 \\
Q - Michaelis-Menten M. & 0,9615 & 28,7544 & 32,19638 & 0,667479 & 0,997997 & 0,5122 \\
R - Weibull & 0,9634 & 30,2933 & 31,72809 & 1,888702 & 0,997974 & 0,4878 \\
\hline Média & 0,9577 & 32,6057 & 33,92647 & 2,133296 & 0,996923 & 0,7012 \\
Desvio-padrão & 0,0236 & 5,9953 & 6,02370 & 1,405397 & 0,001930 & 0,2197 \\
CV\% & 0,0247 & 0,1839 & 0,17755 & 0,658791 & 0,001936 & 0,3133 \\
\hline
\end{tabular}


Rev. Bras. Saúde Prod. Anim., Salvador, v.13, n.1, p.62-73 jan/mar, 2012 http://www.rbspa.ufba.br ISSN 15199940

De acordo com os resultados mostrados nas Tabelas 2, 3 e 4, verifica-se que em média, todos os valores obtidos para o grupo Dorper x Morada Nova (Tabela 2 ), foram melhores do que os dos outros dois grupos. Os ajustes dos modelos para este grupo providenciaram maiores valores para a porcentagem de convergência, $\mathrm{R}_{\text {aj }}^{2}$ e $\mathrm{R}_{\mathrm{p}}^{2}$ e os menores valores para AIC, BIC e MEP. Também se observa que os valores do grupo
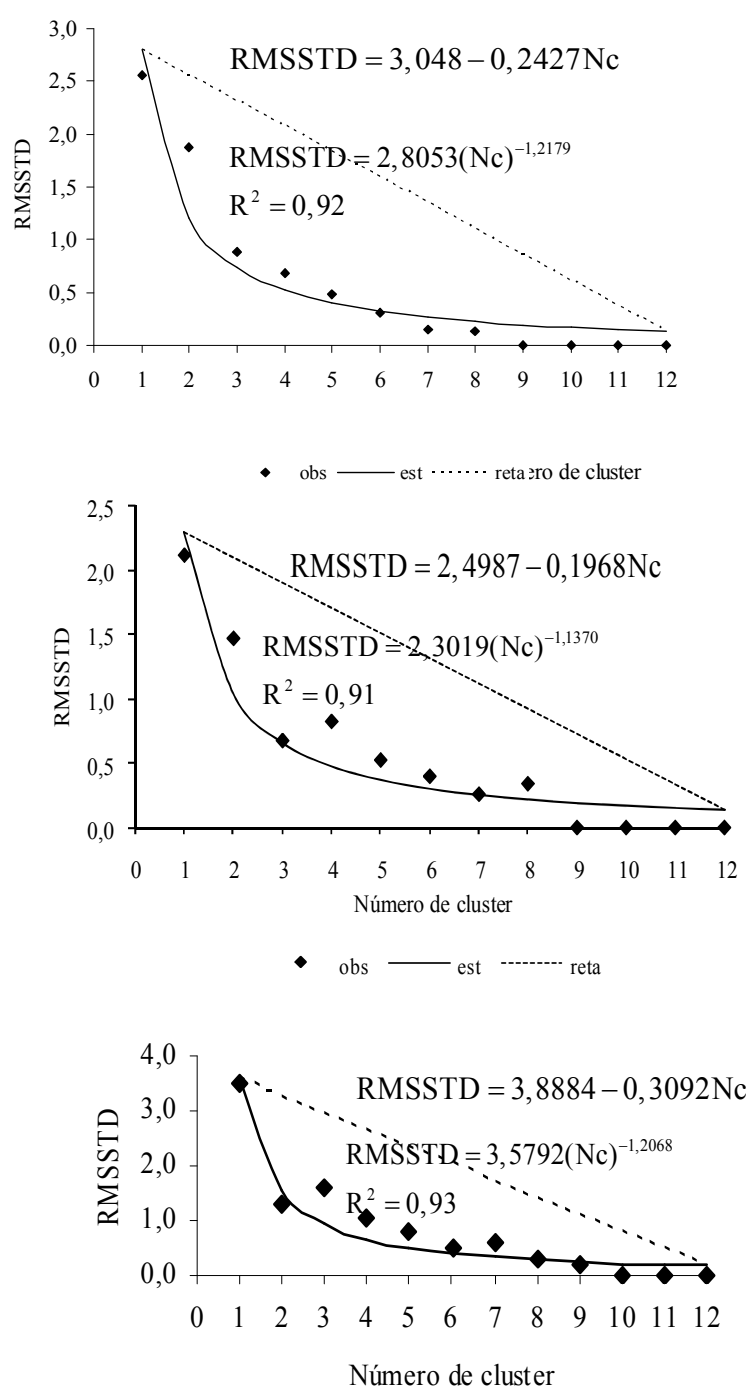

- obs $\longrightarrow$ est $\cdots \cdots \cdot$. reta
Dorper x Santa Inês (Tabela 4) foram melhores que o do grupo Dorper x Rabo Largo (Tabela 3).

Para todos os grupos genéticos avaliados, o número ótimo de cluster obtido por meio da análise da estatística RMSSTD ficou entre três e quatro (Figura 1). Optou-se então por agrupar os 12 modelos em quatro grupos distintos.
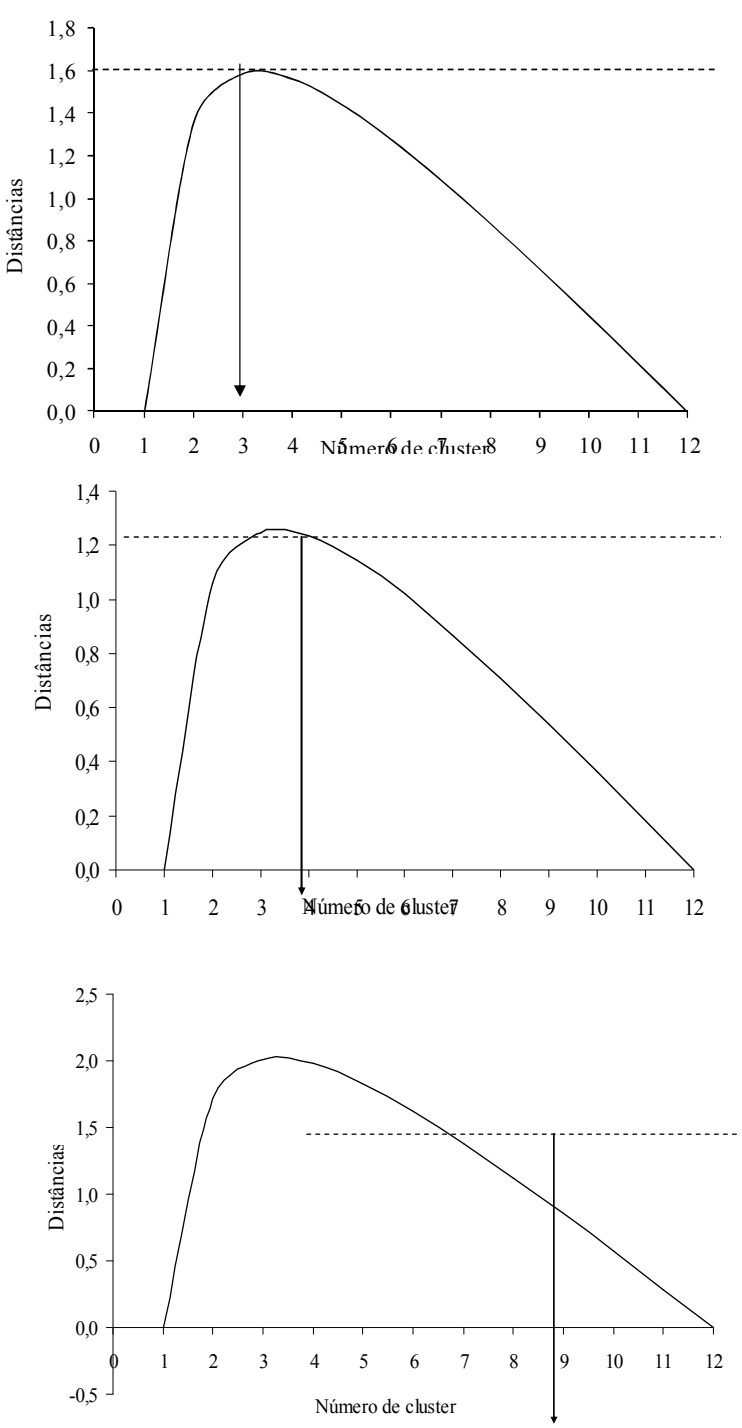

Figura 1. Determinação gráfica do número ótimo de cluster para os grupos genéticos Dorper x Morada Nova (DMN), Dorper x Rabo Largo (DRL) e Dorper x Santa Inês (DSI), respectivamente 
Rev. Bras. Saúde Prod. Anim., Salvador, v.13, n.1, p.62-73 jan/mar, 2012 http://www.rbspa.ufba.br ISSN 15199940

Nas Tabelas 5, 6 e 7 são apresentados os agrupamentos, ordenados pelos valores mais plausíveis para os avaliadores da qualidade de ajuste, com os seus respectivos modelos e médias de tais avaliadores ao considerar o ajuste individual.

Para a ordenação dos agrupamentos, foi dado maior peso para o percentual de convergência ( $\%$ Conv), uma vez que este critério é muito importante em estudos de curvas de crescimento individuais para fins de melhoramento genético. Isto porque, se um modelo apresenta qualidade de ajuste muito alta, mas percentual de convergência muito baixo, o melhorista terá um número reduzido de animais, caracterizados somente por aqueles na qual a convergência foi verificada, para serem utilizados em programas de seleção para eficiência de crescimento medida pelas estimativas dos parâmetros $\beta_{1}$ e $\beta_{3}$.

Tabela 5. Agrupamentos com os seus respectivos modelos e médias dos avaliadores para o grupo genético Dorper x Morada Nova (DMN)

\begin{tabular}{lcccc}
\hline Avaliador & Cluster 1 & Cluster 2 & Cluster 3 & Cluster 4 \\
\cline { 2 - 5 } & Modelos & $\begin{array}{c}\text { Modelos } \\
\{\mathrm{A}\}\end{array}$ & $\begin{array}{c}\text { Modelos } \\
\{\mathrm{Q}\}\end{array}$ & $\begin{array}{c}\text { Modelos } \\
\{\mathrm{C}\}\end{array}$ \\
\hline $\mathrm{R}^{2}{ }_{\text {aj }}$ & 0,9679558 & 0,9536211 & 0,9712000 & 0,9686429 \\
$\mathrm{AIC}$ & 26,7432643 & 36,4340476 & 23,4173077 & 17,0443333 \\
$\mathrm{BIC}$ & 27,6623640 & 37,5127754 & 24,4217272 & 17,4438688 \\
$\mathrm{MEP}$ & 0,9451580 & 2,3941026 & 0,6868304 & 0,3920702 \\
$\mathrm{R}_{\mathrm{p}}^{2}$ & 0,9980098 & 0,9934621 & 0,9983720 & 0,9987439 \\
$\%$ Conv. & 0,6709402 & 0,8076923 & 0,5000000 & 0,2692308 \\
\hline
\end{tabular}

Tabela 6. Agrupamentos com os seus respectivos modelos e médias dos avaliadores para o grupo genético Dorper x Rabo Largo (DRL)

\begin{tabular}{lcccc}
\hline \multirow{2}{*}{ Avaliador } & Cluster 1 & Cluster 2 & Cluster 3 & Cluster 4 \\
\cline { 2 - 5 } & $\begin{array}{c}\text { Modelos } \\
\{\mathrm{D}\}\end{array}$ & $\begin{array}{c}\text { Modelos } \\
\{\mathrm{O}\}\end{array}$ & $\begin{array}{c}\text { Modelos } \\
\{\mathrm{C}, \mathrm{E}\}\end{array}$ & $\begin{array}{c}\text { Modelos } \\
\{\mathrm{F}, \mathrm{N}, \mathrm{H}, \mathrm{B}, \mathrm{P}, \mathrm{Q}, \mathrm{R}, \mathrm{A}\}\end{array}$ \\
\hline $\mathrm{R}^{2}{ }_{\text {aj }}$ & 0,9634389 & 0,9614781 & 0,9693264 & 0,9495330 \\
$\mathrm{AIC}$ & 30,6113000 & 33,1841143 & 26,5922159 & 35,4796137 \\
$\mathrm{BIC}$ & 31,6489212 & 34,2748758 & 27,5727747 & 36,7491817 \\
$\mathrm{MEP}$ & 1,2800661 & 1,2150162 & 1,2951116 & 1,7005855 \\
$\mathrm{R}_{\mathrm{p}}^{2}$ & 0,9968227 & 0,9970889 & 0,9957464 & 0,9962172 \\
$\%$ Conv. & 0,8125000 & 0,7291667 & 0,6145833 & 0,5911458 \\
\hline
\end{tabular}


Rev. Bras. Saúde Prod. Anim., Salvador, v.13, n.1, p.62-73 jan/mar, 2012 http://www.rbspa.ufba.br ISSN 15199940

Tabela 7. Agrupamentos com os seus respectivos modelos e médias dos avaliadores para o grupo genético Dorper x Santa Inês (DSI)

\begin{tabular}{lcccc}
\hline Avaliador & Agrupamento 1 & Agrupamento 2 & Agrupamento 3 & Agrupamento 4 \\
\cline { 2 - 5 } & $\begin{array}{c}\text { Modelos } \\
\{\mathrm{O}\}\end{array}$ & $\begin{array}{c}\text { Modelos } \\
\{\mathrm{P}\}\end{array}$ & $\begin{array}{c}\text { Modelo } \\
\{\mathrm{H}, \mathrm{N}, \mathrm{B}, \mathrm{R}, \mathrm{F}, \mathrm{D}, \mathrm{E}, \mathrm{Q}\}\end{array}$ & $\begin{array}{c}\text { Modelos } \\
\{\mathrm{A}, \mathrm{C}\}\end{array}$ \\
\hline $\mathrm{R}_{\mathrm{aj}}^{2}$ & 0,9782033 & 0,9053306 & 0,9689084 & 0,9289864 \\
$\mathrm{AIC}$ & 24,8735355 & 39,4480229 & 30,0663852 & 43,2078605 \\
$\mathrm{BIC}$ & 25,8071498 & 40,1355319 & 31,4677588 & 44,7164178 \\
$\mathrm{MEP}$ & 1,0606804 & 3,2907039 & 1,5457907 & 4,4409225 \\
$\mathrm{R}_{\mathrm{p}}^{2}$ & 0,9977781 & 0,9947629 & 0,9977799 & 0,9941490 \\
$\%$ Conv. & 0,8536585 & 0,8780488 & 0,6737805 & 0,6463415 \\
\hline
\end{tabular}

Para o grupo Dorper x Morada Nova optou-se pelo agrupamento 1 como aquele que contém os melhores modelos, pelo fato do mesmo apresentar valores plausíveis para os avaliadores e também alto percentual de convergência. Para o grupo Dorper x Rabo Largo destacam-se os agrupamentos 1 e 2 , pois o primeiro apresenta melhores valores para $\mathrm{R}_{\mathrm{aj}}{ }_{\mathrm{j}}$, AIC, BIC, e o segundo para MEP e $\mathrm{R}_{\mathrm{p}}^{2}$, porém optou-se pelo agrupamento 1 por este mostrar maior percentual de convergência. Para o grupo Dorper $\mathrm{x}$ Santa Inês também se destacaram os agrupamentos 1 e 2, e devido a similaridade observada para a percentual de convergência, optou-se pelo primeiro por este apresentar melhores valores para todos os demais avaliadores.

Após a justificativa da escolha dos agrupamentos com os melhores modelos verificou-se que o modelo $\mathrm{O}$ (von Bertalanffy) foi o mais indicado para descrever as curvas de crescimento dos três grupos genéticos, uma vez que está contido no agrupamento 1 , das Tabelas 5 e 7, e no agrupamento 2, da Tabela 6. Em relação à qualidade deste modelo, Guedes et al. (2004) relatam que o mesmo foi o que melhor descreveu curvas de crescimento de cordeiros das raças Santa Inês e
Bergamácia, e Freitas (2005) relata que o modelo em questão, juntamente com o modelo Logístico, são os mais versáteis dentre os modelos de três parâmetros para descrever curvas de crescimento de espécies domésticas.

De acordo com a metodologia proposta neste trabalho, os melhores modelos para o grupo Dorper x Morada Nova foram Mitscherlich, Meloun I e II, Brody, von Bertalanffy, Weibull, Logístico, Gompertz e Michaelis-Menten. Enquanto que para o grupo Dorper x Rabo Largo, $\mathrm{o}$ modelo classificado como o melhor foi o de Gompertz e para o grupo Dorper $\mathrm{x}$ Santa Inês o modelo von Bertalanffy. Vale destacar que a opção por um modelo comum, no caso, von Bertalanffy, ocorre apenas para a aplicação do teste de identidade de modelos.

$\mathrm{Na}$ Tabela 8 são mostrados os resultados referentes aos testes de identidade de modelo, relacionados com as comparações entre as curvas estimadas por meio deste modelo para os três grupos genéticos considerados.

Antes de discutir os resultados da Tabela 8, vale ressaltar que Regazzi \& Silva (2004) recomendam testar a heterogeneidade de variâncias entre os grupos para que a metodologia de identidade de modelos seja válida para 
Rev. Bras. Saúde Prod. Anim., Salvador, v.13, n.1, p.62-73 jan/mar, 2012 http://www.rbspa.ufba.br ISSN 15199940

situação envolvendo repetições, que neste caso são os animais dentro de cada grupo. Sendo assim, o teste de F máximo produziu como resultado $\mathrm{O}$ valor $F=25,7135 / 16,3824=1,5696$, o qual sugere a homogeneidade de variâncias entre os três grupos genéticos estudados.

Tendo em vista os resultados dos testes apresentados na Tabela 8, observa-se que a estimativa do peso adulto para o grupo

Dorper $x$ Santa Inês $\left(\hat{\beta}_{11}=33,2351\right)$ diferiu-se daquela observada para o grupo Dorper x Rabo Largo $\left(\hat{\beta}_{12}=29,2451\right)$, porém não mostrou-se estatisticamente diferente daquela observada para o grupo Dorper x Morada Nova $\left(\hat{\beta}_{11}=31,0107\right)$. Cabe comentar que para a hipótese que envolveu os três grupos, $\mathrm{H}_{0}^{(1)}$, embora não se tenha constatada a significância ao nível de $5 \%$ de probabilidade, os valores das estatísticas $\chi_{\text {calculado }}^{2} \chi_{\text {Tabelado foram }}^{2}$ muito próximos, portanto assumiu-se a rejeição da hipótese em questão, fato este que culminou nas diferenças significativas apresentadas.

Tabela 8. Testes de identidade de modelos tendo em vista os parâmetros do modelo von Bertalanffy que apresentam interpretação biológica $\left(\beta_{1 \mathrm{k}} \text { e } \beta_{3 \mathrm{k}}\right)^{(1)}$

\begin{tabular}{|c|c|c|c|c|c|}
\hline Hipóteses & $\mathrm{n}$ & $\begin{array}{c}\mathrm{SQR}_{\Omega} \\
\text { (Completo) }\end{array}$ & $\begin{array}{c}\mathrm{SQR}_{\omega} \\
\text { (Reduzido) }\end{array}$ & $\chi_{\text {Calculado }}^{2}$ & $\chi_{\text {Tabelado }}^{2}$ \\
\hline $\mathrm{H}_{0}^{(1)}: \beta_{11}=\beta_{12}=\beta_{13}$ vs $\mathrm{H}_{\mathrm{a}}^{(1)}$ & 987 & 19300,4 & 19407,6 & $5,4669^{\mathrm{ns}}$ & $\begin{array}{c}\chi_{5 \%}^{2}(2)= \\
5,9915\end{array}$ \\
\hline $\mathrm{H}_{0}^{\left(1_{\text {DMNXDRLL }}\right)}: \beta_{11}=\beta_{12}$ vs $\mathrm{H}_{\mathrm{a}}^{\left(1_{\text {DMNXXRL }}\right)}$ & 608 & 9632,1 & 9637,9 & $0,3659^{\mathrm{ns}}$ & \multirow{3}{*}{$\begin{array}{c}\chi_{5 \%}^{2}(1)= \\
3,8415\end{array}$} \\
\hline $\mathrm{H}_{0}^{\left(l_{\text {DMNXDII }}\right)}: \beta_{11}=\beta_{13}$ vs $\mathrm{H}_{\mathrm{a}}^{\left(\mathrm{l}_{\mathrm{DMN} \times \mathrm{DSI}}\right)}$ & 599 & 12993,2 & 12999,7 & $0,2999^{\mathrm{ns}}$ & \\
\hline $\mathrm{H}_{0}^{\left(\mathrm{l}_{\mathrm{DRL} L \mathrm{DSI} I}\right)}: \beta_{12}=\beta_{13} v s \quad \mathrm{H}_{\mathrm{a}}^{\left(\mathrm{l}_{\mathrm{DRLXDSI}}\right)}$ & 767 & 15975,5 & 16082,0 & $5,0962^{*}$ & \\
\hline $\mathrm{H}_{0}^{(3)}: \beta_{31}=\beta_{32}=\beta_{33}$ vs $\mathrm{H}_{\mathrm{a}}^{(3)}$ & 987 & 19300,4 & 19338,6 & $1,9515^{\mathrm{ns}}$ & $\begin{array}{c}\chi_{5 \%}^{2}(2)= \\
5,9915\end{array}$ \\
\hline
\end{tabular}

${ }^{1} \mathrm{O}$ índice k corresponde aos três grupos genéticos: $\mathrm{k}=1$ Dorper x Morada Nova (DMN), 2 Dorper x Rabo Largo (DRL) e 3 Dorper x Santa Inês (DSI).

*Significativo ao nível de $5 \%$ de probabilidade.

Em relação ao peso adulto, os resultados observados concordam com aqueles obtidos por Carneiro et al. (2007), os quais estudaram curvas de crescimento individuais destes mesmos grupos por meio do modelo Logístico e também observaram maior peso adulto para o Dorper x Santa Inês, precedido do Dorper x Morada Nova e do Dorper $\mathrm{x}$ Rabo Largo, cujas estimativas foram respectivamente $28,6809,27,1443$ e 26,0857 .
Quanto ao teste da hipótese $\mathrm{H}_{0}^{(3)}$, relacionada com a taxa de maturidade, nota-se que a mesma não apresenta indícios de ser rejeitada, fato este que nos permite inferir que os três grupos genéticos estudados não diferem em relação a este parâmetro, cujas estimativas foram $0,0090,0,0113 \mathrm{e}$ 0,0119 , respectivamente para os grupos Dorper x Morada Nova, Dorper x Rabo Largo e Dorper x Santa Inês. Diante do explícito, não se justifica apresentar os testes de hipóteses referentes 
às comparações duas a duas como realizado para o parâmetro peso adulto.

De forma geral, os resultados apresentados e discutidos, nos permitem concluir sob o ponto de vista zootécnico, que o grupo genético Dorper x Santa Inês é o mais indicado para se explorar a produção de carne, tendo em vista as condições experimentais do presente estudo. Tal conclusão está fundamentada no fato deste grupo ter apresentado o maior peso adulto $\left(\beta_{1}\right)$, uma vez que na atual ovinocultura de corte do Nordeste brasileiro ainda não se tem incentivo para o abate de animais precoces, aqueles com maior taxa de maturidade $\left(\beta_{3}\right)$, os quais, embora sejam menores, apresentam melhor qualidade de carne. Em resumo, no atual sistema de produção preconiza-se a quantidade de carne, e não a sua qualidade (MALHADO et al., 2009).
A Figura 2 mostra as curvas de crescimento estimadas para cada grupo genético, inclusive com a visualização de uma região de extrapolação que permite constatar as diferenças significativas dos testes de identidade de modelo. Tal região foi considerada devido ao fato dos dados observados não contemplarem idades avançadas, nas quais se podem observar os valores estimados para o peso adulto. Por meio desta Figura nota-se que o peso adulto referente ao Dorper $\mathrm{x}$ Santa Inês realmente é maior que aquele referente ao grupo Dorper x Rabo Largo, e que o peso adulto apresentado pelo grupo Dorper x Morada Nova caracteriza-se como um valor intermediário, não sendo, portanto, estatisticamente diferente de nenhum dos demais.

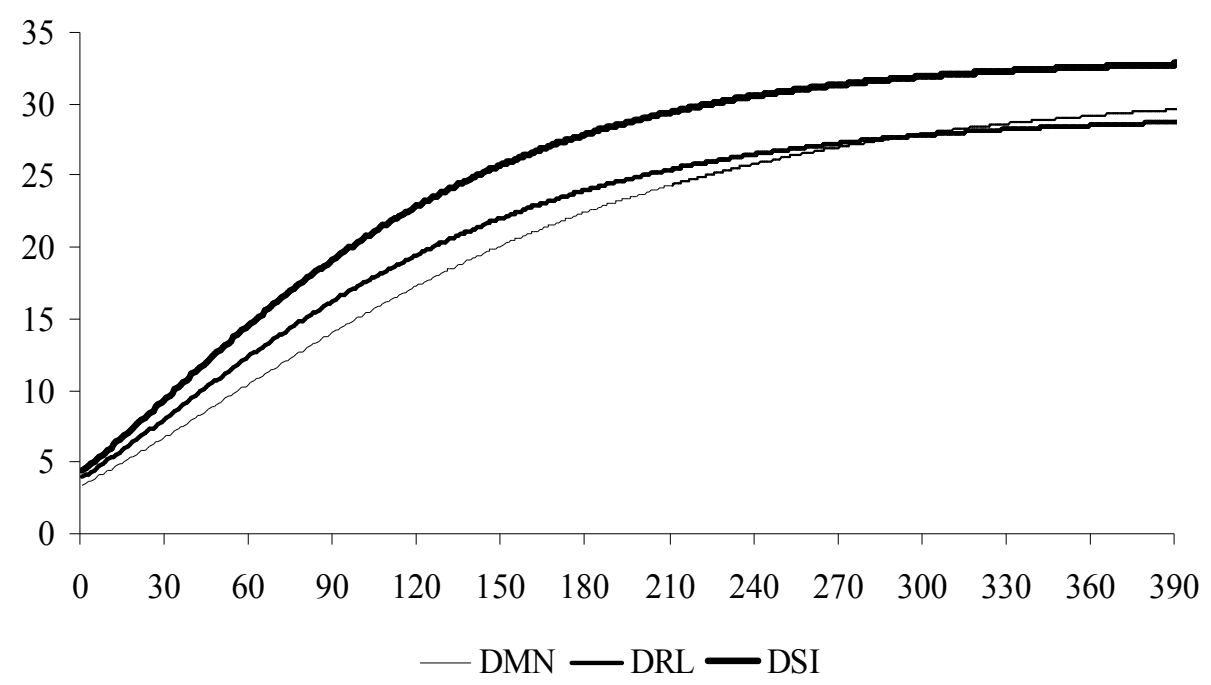

Figura 2. Curvas de crescimento estimadas para os grupos genéticos Dorper x Morada Nova (DMN), Dorper x Rabo Largo (DRL) e Dorper x Santa Inês (DSI)

A metodologia proposta no presente trabalho mostrou-se adequada para classificar modelos não-lineares tendo em vista a escolha de um único e melhor modelo para descrever o crescimento dos três grupos genéticos estudados. O modelo von Bertalanffy é o que apresenta os melhores ajustes para o conjunto de dados individuais. De acordo com testes de identidade de 
Rev. Bras. Saúde Prod. Anim., Salvador, v.13, n.1, p.62-73 jan/mar, 2012 http://www.rbspa.ufba.br ISSN 15199940

modelos, o grupo genético Dorper $\mathrm{x}$ Santa Inês é o que apresenta maior peso adulto, portanto, este, o mais recomendado para exploração de carne.

\section{REFERÊNCIAS}

BARROS, N.N.; VASCONCELOS, V.R.;WANDER, A.E.; ARAÚJO, M.R.A. Eficiência bioeconômica de cordeiros F1 Dorper $\times$ Santa Inês para produção de carne. Pesquisa

Agropecuária Brasileira, v.40, p.825831, 2005.

CARNEIRO, P.L.S.; MALHADO, C.H.M.; SOUZA JÚNIOR, A.A.O.; SILVA, A.G.S.; SANTOS, F.N.; SANTOS, P.F.; PAIVA, S.R. Desenvolvimento ponderal e diversidade fenotípica entre cruzamentos de ovinos Dorper com raças locais. Pesquisa Agropecuária Brasileira, v.42, n.7, p.991-998, 2007.

CECON, P.R.; SILVA, F.F.; FERREIRA, A.; FERRÃO, R.G.; CARNEIRO, A.P.S.; DETMANN, E.; FARIA, P.N.; MORAIS, T.S.da.S. Análise de medidas repetidas na avaliação de clones de café 'Conilon'.

Pesquisa Agropecuária Brasileira, v.43, n.9, p.1171-1176, 2008.

FREITAS, A.R. Curvas de crescimento na produção animal. Revista Brasileira de Zootecnia, v.34, n.3, p.786-795, 2005.

GUEDES, M.H.P.; MUNIZ, J.A.; PEREZ, J.R.O.; SILVA, F.F; AQUINO, L.H.; SANTOS, C.L. Estudo das curvas de crescimento de cordeiros das raças Santa Inês e Bergamácia considerando heterogeneidade de variâncias. Ciência e Agrotecnologia, v.28, n.2, p.381-388, 2004.
MALHADO, C.H.M.; CARNEIRO, P.L.S.; AFFONSO, P.R.A.M.; SOUZA Jr., A.A.O.; SARMENTO, J.L.R.

Growth curves in Dorper sheep crossed with the local Brazilian breeds, Morada Nova, Rabo Largo, and Santa Inês.

Small Ruminant Research, v.84, p.1621, 2009.

MALHADO, C.H.M.; CARNEIRO, P.L.S.; SANTOS, P.F.; AZEVEDO, D.M.M.R.; SOUZA, J.C.; AFFONSO, P.R.M. Curva de crescimento em ovinos mestiços Santa Inês x Texel criados no Sudoeste do Estado da Bahia. Revista Brasileira de Saúde e Produção Animal, v.9, n.2, p. 210-218, 2008.

REGAZZI, A.J.; SILVA, C.H.O. Teste para verificar a igualdade de parâmetros e a identidade de modelos de regressão não-linear. I. Dados no delineamento inteiramente casualizado. Revista Matemática e Estatística, v.22, n.3, p.33-45, 2004.

SAS INSTITUTE. Statistical Analysis System user's guide. Version 9.1. Cary, USA, 2003.

SILVEIRA, F.G. Classificação multivariada de modelos de crescimento para grupos genéticos de ovinos de corte. 2010. 61f. Dissertação (Mestrado em Estatística Aplicada e Biometria) - Universidade Federal de Viçosa, Viçosa.

Data de recebimento: 29/06/2011

Data de aprovação: 09/02/2012 